“ (C) 2018 IEEE. Personal use of this material is permitted. Permission from IEEE must be obtained for all other uses, in any current or future media, including

reprinting/republishing this material for advertising or promotional purposes, creating new collective works, for resale or redistribution to servers or lists, or reuse of any copyrighted component of this work in other works." 


\title{
SAM Smart Asthma Monitoring: Focus on Air Quality Data and Internet of Things (IoT)
}

\author{
Nikita Isaac, Naveena Sampath and Valerie Gay \\ Faculty of Engineering and IT \\ University of Technology Sydney \\ Broadway NSW 2007, Australia \\ Email: Valerie.Gay@uts.edu.au
}

\begin{abstract}
Nowadays, mobile technologies and in particular the Internet of Things (IoT), data and machine learning technologies can have a positive impact on the way we manage our health. This paper focuses on Asthma, a condition that affects $10 \%$ of Australians. This research collects and analyses data about air quality using New South Wales regional area data and data obtained from our faculty smart building (UTS building 11). Based on a persons location and travel plans, we analyze the Air Quality Index (AQI) and provide a personalized and localized feedback to individuals living with asthma and help them manage their condition better.
\end{abstract}

Keywords-Asthma monitoring; mobile technology; wearable technology; machine learning; data analysis; personalised feedback;

\section{INTRODUCTION}

According to The Global Asthma Report 2014, more than 334 million people have asthma worldwide [1]. In Australia, approximately $10 \%$ of the population suffers from the disease [2]. For many people living with asthma, the onset of symptoms is a direct result of environmental factors, or factors relating to the individual's physical condition. In the United States, asthma has seen an increased prevalence of asthma in all ages, from $7.3 \%$ (20.3 million people) to $8.2 \%$ (24.6 million people) over the last decade [3]. In the United States, asthma is the most common chronic disease among youth [4] and carries an economic burden of $\$ 56$ billion [5]. According to the Australian Bureau of Statistics (ABS), Asthma affects approximately 2.3 million people in Australia with $\$ 655$ million spent on asthma in 2008-2009; or approximately $0.9 \%$ of all direct health spending on diseases [6].

As the prevalence of asthma is increasing, and with no known cure, there needs to be further research into how the disease can be managed and controlled more effectively. Studies have shown that there are several environmental and physical triggers to asthma. The environmental factors that are known to trigger or exacerbate asthma symptoms are extreme temperatures, extreme humidity or dryness, air pollution, pollen, smoke, and mould [7,8]. However, the effect of these environmental factors varies on a per patient basis. There is also a relationship between physical activity and asthma that can impact daily living [9]. Cardiorespiratory performance of asthmatic patients is suboptimal, but medically supervised physical activity can produce beneficial results for those who have asthma. Insufficient sleep and poor sleep hygiene can lead to increased asthma symptoms the following day, and Asthma also affects sleep [10].
The top factors contributing to asthma susceptibility were factors based on physical activity and work stress [11] [10]. Some popular examples of wearable fitness tracking devices are Fitbit, Garmin, and Jawbone. Data can also be tracked directly within a smartphone, such as the GPS location and manual input, and aggregated with additional data such as weather forecast. Web pages such as www.weatherzone.com.au allow for developers to access these attributes via their API. The increased adoption of wearable fitness tracking devices acquires the subsequent metadata to assist asthma sufferers proactively manage their condition.

Air Quality Index (AQI) is the term used by the authorities to express recent air quality and to provide a prediction of air quality. Using environmental data available on the cloud, wearable fitness tracking devices, building sensory equipment, peak flow meter data, and location-specific information, our architecture presents how asthma can be monitored with tailored feedback and up-to-date asthma management plan for individuals and empower asthma sufferers by giving them relevant information when needed.

The Internet of Things (IoT) has enabled buildings and homes to be more connected [12]. At UTS building 11, building sensors are typically used to determine occupancy levels and energy efficiency [12]. Some of the indoor building conditions such as oxygen levels, smoke detection, temperature, humidity and levels of indoor air pollutants can be obtained from these building sensors, which have a direct influence on asthma [13]. Building sensors can be useful in assessing exposure and identifying environments that are causing asthma attacks both short and long-term.

There are two types of asthma such as asthma caused by allergens, and asthma caused by air quality, stress, physical activity and weather conditions [2]. Air quality is one of the triggers of asthma that is caused due to the presence of air pollutants in the air. However, some asthma patients have varying levels of tolerance to the triggers mentioned above [3] [4]. Elevated levels of Ozone $\left(\mathrm{O}_{3}\right), \mathrm{PM}_{2.5}$, and $\mathrm{PM}_{10}$ also cause long-lasting lung diseases in people living with asthma [5]. Web pages such as www.environment.nsw.gov.au provide expected air quality for the regional areas of NSW. In Australia, bushfires instigate an adverse impact on air quality. Air quality is typically elevated in the presence of smoke and particulate materials. This is the reason asthma sufferers need attention while they are outdoor [11]. Various surveys and studies are conducted through social media such as Facebook, Twitter to investigate the degree of people living with asthma in Australia [8]. With the help of the 
analysed data, about $75 \%$ of asthma patients are shown using broadcasting forewarns to change their outdoor activities [9].

The sensors are embedded within electronic devices to detect the air quality, humidity and weather, thereby alerting the users whether to stay indoors or outdoors [31]. There are also sensors that detect the number of times inhalers are used by the patients, therefore keeping track of their inhaler usage. The Smart Peak Flow device is a recently developed device for smartphones that has a propeller that rotates when the user blows and connects the reading with the smartphone application [28]. AQI indications on smartphones are on research, but the other applications such as smart peak flow are high in use. The mobile interfaces have been developed to connect Global System for Mobile communications (GSM) with the cell identities of the users to show the pollution reports to the users $[29,30]$.

There are currently some asthma management smartphone apps available, including AsthmaMD, which allow users to manage their asthma and record vital information including medication, action plans and reminder systems [14]. Many of these apps require the manual input of data, such as peak flow measurements, height, weight, and age, then predict the risk of acute asthma symptoms occurring based on this data $[15,16,17]$.

There are a considerable amount of projects that currently focus on asthma management. However, these plans focus on providing notifications to the users using sensors that can only function efficiently in a regulated environment. If the individual is present in a pervasive environment, their performances are limited, and air quality is overlooked in these asthma management applications (For instance, Propeller - Measures inhaler usage, Asthma Buddy - Traces variations in symptoms).

In this paper, we propose an architecture for smart asthma management. It collects data using Microsoft Excel and analyses data using MATLAB, thereby gives feedback to provide localized and personalised smart asthma monitoring.

In section 1, the paper presents a Smart Asthma Monitoring (SAM) architecture. In Section 2, it discusses the data collected from multiple sources and their aggregation. In Section 3 , it focuses on the data analysis and section 4 on the feedback. The conclusion summarises our focus on using the personalised feedbacks to make the necessary changes such as while the individuals are in the indoor environment. The paper is based on our current research work on air quality in the NSW regional areas, UTS building 11 and mobile personalised health monitoring.

\section{ARCHITECTURE}

Our proposed architecture for SAM is presented in figure 1. It uses an App and cloud services to collect and aggregate the data relevant to the individual, analyse the data using both machine learning and health professional expertise and provide personalised feedback. The feedback includes reminders such as regular medications, upcoming sports activity. The feedback also includes localized information about asthma triggers such as pollution levels and shows the high-risk locations that have triggered asthma attacks for them or even people like them. These feedbacks make it easier for users to keep track of their asthma. The architecture could warn hospital of the need for extra hospital beds; it could even deliver the medicine, pre-emptively change the medication and act on the smart environment (close car windows and start the air purifier).

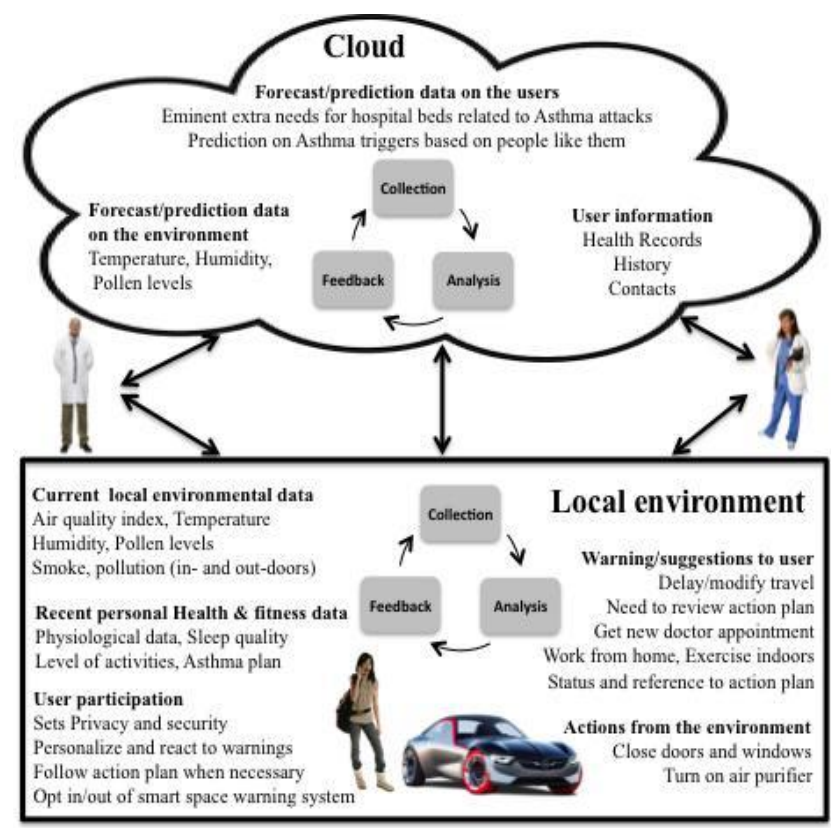

Fig. 1. Smart Asthma Monitoring Architecture

\section{COLLECTION AND AGGREGATION}

By embedding the air quality sensors with computing software such as MATLAB, IoT technology enhances the accuracy and efficiency by providing feedback to the individuals through smart devices based on the analysed data. This allows to connect and interact with the data.

Microsoft Excel spreadsheets are considered beneficial for data collection, especially when the collected data is used for data analysis. Excel as a data collection tool can retrieve definite information regardless of user interference. Data collection and storage is done certainly where the data cleaning is more accessible in excel compared to other data collection tools [23].

The indoor environment settings such as Oxygen $(\mathrm{O} 2)$, Carbon dioxide (CO2), Hydrocarbons (HO), dust particles and air pollutants are attained from the building sensors of UTS building 11 that help with indicating the conditions that should be avoided for people living with asthma. The outdoor AQI in New South Wales (NSW) is composed of O3, Nitrogen dioxide (NO2), Visibility (NEPH), Carbon monoxide (CO), Sulphur dioxide (SO2), PM2.5 and PM10 [25] which are obtained from the sensors installed by NSW Office of Environment and Heritage. 
Data collection is done using Microsoft Excel for a userspecified period. For the outdoor environment, the data is collected between the time specified by user. The user can select the location for which they require the personalised feedbacks. The users can also select the time frame and exact time for which they require the personalised feedbacks. The sensors collected the data continuously and based upon the data collection through Microsoft excel. Upon the user's selection of the date, time and location, MATLAB analysis the data and accordingly the feedbacks are provided to the users.

For instance, if 4.00 PM to 5.00 PM is selected since it is the peak time involving heavy transports and a substantial number of people are travelling outdoors, the same time and dates can be followed for the indoor environment and case study as well.

1. The data collected for outdoor environment is gathered from the website http://www.environment.nsw.gov.au/

2. The data collected for indoor environment is inferred from the website https://eif-research.feit.uts.edu.au/, where the data is obtained from the building sensors of UTS building 11.

Data cleaning is not necessary for the outdoor environment. However, for the indoor environment, a huge volume of data is recorded from the sensors. Therefore, the excel functions such as $=\max (x: y)$ and $=\min (x: y)$ are used for data cleaning. For the indoor environment, the data that is obtained from the sensors are raw data except for air pollutants and hence they are standardized to parts per million ( $\mathrm{ppm}$ ) for $\mathrm{CO} 2, \mathrm{HO}$, and percentages for $\mathrm{O} 2$.

On a typical day, SAM collects the air quality data from the indoor, outdoor environment and the location of travel, to provide personalised feedback to the individuals. The user controls the feedbacks of the location. That is, for instance if the user wants to obtain feedback on air quality from Randwick to Rozelle, she can get that required feedback. The user is in control of the data she wants to collect locally and whether she wishes to share it or not and with whom. She can enter data manually and annotate a reading by adding comments and contextual information, such as extraperformed activities. SAM can also obtain data from sensors and wearable around the user and in the cloud.

Quality of the data obtained from the various sources may not always be accurate and reliable. It is therefore important to tag the data collected and take the data quality into account when deciding. The required quality and source of the data are dependent on the individual patient's susceptibility. Once a patient has developed a historical data pattern, this can be used to determine the quality of the attribute relative to that person.

\section{DATA ANALYSIS}

Previously, AQI has been calculated mathematically, which is currently replaced by data analysis practices [22]. The data analysis is done using MATLAB, which analyses the collected data from outdoor regional areas of NSW and the indoor UTS buildings that are embedded to provide the personalized feedback to the individuals. MATLAB is a mathematical, analytical software that can handle processing of real-time data using microcontrollers such as Arduino UNO and electronic sensors. Data analysis with MATLAB software is efficient since excel can be read into MATLAB and Matlab handles a high volume of data with lesser delay [24].

Data analysis is done using MATLAB. The data from Microsoft excel are read into MATLAB using the function

“aqi=xlsread('Excel_filename,'sheet_number')". For instance, to read the AQI data of Randwick region, the following function is used,

Randwick=xlsread('Excel_filename','sheet_number,'cell_nu $\underline{\text { mber'); }}$

Upon data analysis, IoT helps the users directly connect with the environment they belong in and provides personalized feedback on the air quality in their location. The users can select their means of storage, whether on their mobile devices or on the cloud. Depending on the history of storage, feedbacks can regularly be provided.

In addition to that, various other conditional functions are used to compare the collected data to the threshold levels. In case of the indoor environment, there are different conditions for different elements which are detailed in the results section. Based on the analysis, some of the outdoor regions have frequently been identified to have an elevated AQI level. While some outdoor regions are marked safe for most of the time. The cases are detailed in the result section.

Table I

\begin{tabular}{|c|c|}
\hline AQI & Personalised feedback \\
\hline $0-33$ & Very Safe to travel Outdoor \\
\hline $34-64$ & Safe to travel Outdoor \\
\hline $65-99$ & Sensitive people advised staying \\
& indoors \\
\hline $100-149$ & $\begin{array}{c}\text { Poor air quality. Sensitive people } \\
\text { highly advised to stay indoors }\end{array}$ \\
\hline $150-199$ & $\begin{array}{c}\text { Warning levels of air quality. Sensitive } \\
\text { people must stay indoor }\end{array}$ \\
\hline 200 & $\begin{array}{c}\text { Hazardous levels of air quality. } \\
\text { Sensitive people must avoid outdoor } \\
\end{array}$ \\
& activities \\
\hline & a. Conditions for outdoor air quality analysis \\
\hline
\end{tabular}


The air quality analysis for the outdoor environment is done based on the above conditions. For an outdoor location showing the air quality index obtained from the website NSW Office of environment and heritage, the above feedbacks will be provided to the users upon their selection of location.

Table II

\begin{tabular}{|c|c|c|}
\hline $\begin{array}{l}\text { Indoor } \\
\text { elements }\end{array}$ & $\begin{array}{l}\text { Quantity } \\
\text { present } \\
\text { indoor }\end{array}$ & Personalised feedback \\
\hline \multirow{3}{*}{$\begin{array}{l}\text { Carbon } \\
\text { dioxide }\end{array}$} & $\begin{array}{l}380 \mathrm{ppm} \text { to } \\
398 \mathrm{ppm} \\
\end{array}$ & Ventilation is required. \\
\hline & $\begin{array}{|ll|}399 & \mathrm{ppm} \\
\text { and above } & \\
\end{array}$ & $\begin{array}{l}\text { Immediate } \\
\text { required. }\end{array}$ \\
\hline & \begin{tabular}{|l|}
$360 \quad \mathrm{ppm}$ \\
and below
\end{tabular} & $\begin{array}{l}\text { The area is well- } \\
\text { ventilated. }\end{array}$ \\
\hline \multirow{4}{*}{ Hydrocarbons } & $\begin{array}{l}35 \mathrm{ppm} \text { to } \\
399 \mathrm{ppm}\end{array}$ & $\begin{array}{c}\text { People sensitive to } \mathrm{CO} \\
\text { concentration need } \\
\text { attention } \\
\end{array}$ \\
\hline & $\begin{array}{l}\text { 400ppm } \\
\text { and above }\end{array}$ & $\begin{array}{l}\text { Dangerous levels of } \\
\text { carbon monoxide. }\end{array}$ \\
\hline & $\begin{array}{c}10 \mathrm{ppm} \text { to } \\
15 \mathrm{ppm}\end{array}$ & Concentration is safe \\
\hline & 0 to $9 \mathrm{ppm}$ & Concentration is safest. \\
\hline \multirow[b]{2}{*}{ Oxygen } & Below $21 \%$ & Area requires ventilation \\
\hline & $\begin{array}{l}21 \% \quad \text { and } \\
\text { above }\end{array}$ & \begin{tabular}{lll|} 
Area is & properly \\
ventilated & & \\
\end{tabular} \\
\hline \multirow{3}{*}{ Air pollutants } & 0.8 to 1.0 & Air is clean. \\
\hline & Below 0.8 & $\begin{array}{l}\text { Dangerous gases may be } \\
\text { present. }\end{array}$ \\
\hline & Above 1.0 & $\begin{array}{l}\text { Air is clean, but a } \\
\text { negligible amount } \\
\text { pollutants may be present. }\end{array}$ \\
\hline \multirow[t]{2}{*}{ Dust Particles } & $\begin{array}{ll}0.1 & \text { and } \\
\text { Below } & \\
\end{array}$ & $\begin{array}{l}\text { No dust particles } \\
\text { present }\end{array}$ \\
\hline & Above 0.1 & $\begin{array}{l}\text { Dust particles are present. } \\
\text { Area needs attention. }\end{array}$ \\
\hline
\end{tabular}

b. Conditions for indoor air quality analysis

The above table consists of the data analysis conditions for the indoor environment. For analyzing the air quality of indoor environment, we selected the location ES_B_08_422_7BDC. The area is selected as many students use the area for studying, and there are various labs and staff rooms in that location. There are various sensors at various levels at UTS building 11. For instance, ES_A_13_276_7C44 location has dust particles detecting sensors to distinguish the clean air and dust particles such as cigarette smokes.

If the user chooses to store data, depending upon selection data can be stored locally on the mobile device, on the cloud or on a server chosen by the user. The user opts for this storage option to get more intelligent feedbacks based on his long-term history and possibly comparing it to other citizens with similar conditions. Some users will accept that to get better insights while others will opt for less feedback and keep their data private.

These outdoor AQI feedbacks when provided to the hospitals and government health experts, they can arrange the hospitalization facilities and medical treatments in that location if suddenly a high AQI break-out is prominent.

Users may also opt for his clinicians to get access to their data and decide, for example, that there is a need to adjust their asthma plan. This option gives clinicians an overall view of their patient health and fitness data. This enables them making a more precise and personalised valuation. This could also allow the hospital to estimate how many hospital beds they need to reserve for victims of an asthma attack.

Once the data is aggregated, it is filtered it into standard data sets with the required attributes for the individual. The rule-based engine, which is effectively a decision-making tree performs classification of the data. With that data, we can anticipate the likelihood of a flare up and inform the user accordingly.

On the cloud, machine learning methods are used to classify the collective data into sub-sections based on rule engine's inbuilt decision-making criteria. Machine learning can not only help classify asthma factors and management but also can be used in understanding more factors that may trigger an asthma attack, the level of influence it may have on the patient and the relationship between these factors and emergency department visits. Classification and Regression Trees (CART) is a tree building technique and is used in generating clinical decision rules [18].

An important aspect of SAM is that health professionals are not left out of the system. They are important players in the data analysis and the personalized feedback provided to the system and to its users.

\section{FEEDBACK}

For the feedback, we opted a user-centred approach where the user is in control of the way the data is presented to him. Triggers are personalized to the user and the app provides a threshold for triggering an alert. For example, the app would alert a user when the air quality deteriorates and raise the alarm if it gets hazardous. The user can either see the data in real-time, or just get a notification or nudge when necessary or even get a phone call from his doctor if he needs to do something. Users can opt for a nudge when needed and an automatic reaction from his smart space when possible (automatic closing/opening of doors and windows at home or in the car, adjustment of the air conditioning. They can select to have real-time analysis and get warnings and references to their asthma plan based on their personal threshold levels on several parameters (including pollen and pollution level, peak flow readings). Users may also be contacted by their clinicians to make an appointment or to adjust their asthma plan. Based on calendar information that contains the place and activities the user is planning to go to, they may also receive some advice to change plans (e.g.: work from home, exercise indoor) based on the location's current and forecasted data.

The regions that more regularly show elevated pollution levels are Chullora, Randwick, Macquarie Park, Camden, Bringelly, Richmond, Campbelltown, Muswellbrook, and Illawarra region. The regions that are always safe to stay outdoor are a Lower hunter (Wallsend, Newcastle, and Beresfield), Central Tablelands (Bathurst), North-west (Tamworth) and South-West slopes (Albury, Wagga Wagga North) and Singleton. It can also be observed that, with the 
increase in temperature, the air quality index is also increasing. Therefore, the air quality index is higher on sunny days when compared to the other days. This feedback depends on the days and time. IOT allows its servers to remotely and locally store the information, thereby providing a record of their routine activities.

The feedback also addresses one of the challenges: to identify the data that is important for a user. Part of the feedback from the data analysis can be to change the data collection (quality- or quantity- wise) based on previous flare-ups or patients like the users.

SAM can provide personalized feedback to the individuals suffering from asthma based on their location and area of travel. IoT when embedded with the smart devices of the users can direct the feedbacks whether the users should stay indoors, travel outdoor or avoid direct exposure.

Case study is considered at the same time from the location Randwick to Rozelle. Upon their corresponding AQI the following feedbacks are provided. The air quality feedback for asthma plan are provided in the following MATLAB output,

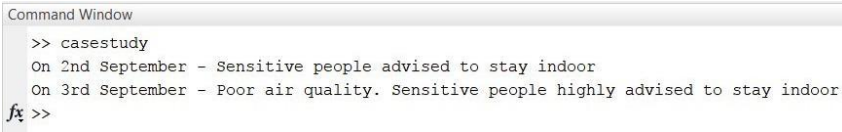

Fig. 2. MATLAB output displaying personalised feedback

SAM uses the results of the data analysis and the help of health professionals to update the individual's asthma management plan or determine whether the user needs to take any action to address their asthma. SAM users do not need to refer to their asthma plan all the time manually; if they opt for it, the app will update them when they need to act.

\section{DISCUSSION AND CONCLUSIONS}

This paper portrayed our vision of how life can be improved for asthma sufferers through the development of a personalized smart asthma monitoring with a focus on air quality analysis. The portrayal is based on the collected data samples and their analysis.

We aim to make life easier for those who suffer from asthma by creating tailored feedback and personalized up-to-date asthma management plans using readily available data and machine learning. Today, as an asthma sufferer, people carry around multiple medications, inhaler spacer and sensors like a peak flow meter and it is hard to keep track of everything. Our proposed architecture uses an App and cloud services to collect and aggregate the data relevant to the individual, analyze the data and provide timely and personalized feedback. In the near future, it could even deliver the medicine, pre-emptively change the medication and act in the smart environment.
The personalised feedbacks can be based on the individuals' location of travel. The feedbacks which can either be suggestions or warnings to the individuals can advise them to stay indoors based on the AQI level, advice the individuals to close their windshields while travelling through the AQI elevated locations or provide the indoor ventilation levels and air quality levels in UTS building 11.

Several areas require further research such as using mathematical methods for data cleaning. The areas that do not require further implementation include collection of data in software, since they do not focus on real-time feedback. The future scope of the project includes, using various data technologies such big data technologies and machine learning. Therefore, real-time data can be processed and analysed to provide contemporary feedback to the individuals suffering from asthma. This development of asthma plans when embedded with smart systems such as smart home, smart transport and smart intelligent systems can provide a greater benefit for the asthma sufferers, thereby enabling them to lead a healthier life.

SAM reminds asthma sufferers to take regular medications and informs asthma triggers such as high pollen count depending upon the locations. This makes asthma management easier, smarter and the technology is here today. This could decrease unnecessary visit to the emergency department, as most of hospital visits are preventable with better asthma management. SAM empowers asthma sufferers with their routine activities and helps health professionals to monitor and control asthma in an informed way.

\section{REFERENCES}

[1] The Global Asthma Network. (2014). The Global Asthma Report. Auckland, New Zealand.

[2] Statistics - An Asthma Australia site. (2016). Asthmaaustralia.org.au. Retrieved 25 February 2017, from http://www.asthmaaustralia.org.au/national/about-asthma/what-isasthma/statistics

[3] Centers for Disease Control and Prevention. Vital signs: asthma prevalence, disease characteristics, and self-management education United States 2001-2009. Morb Mortal Wkly Rep Surveill Summ. 2011; 60(17):546-52.

[4] World Health Organization. Asthma Fact Sheet. 2011. Report No.: N307.

[5] Cleland, J. C., Jan; Ryan, Dermot. (2007). A qualitative study of the attitudes of patients and staff to the use of mobile phone technology for recording and gathering asthma data. Journal of Telemedicine and Telecare, 13, 85-89.

[6] Profiles of Health, Australia, 2011-13. (2016). Abs.gov.au. Retrieved 25 February 2017, from

http://www.abs.gov.au/ausstats/abs@.nsf/Lookup/4338.0main+featur es152011-13

[7] CDC - Asthma - Common Asthma Triggers. (2012). Cdc.gov. Retrieved 25 February 2017, from http://www.cdc.gov/asthma/triggers.html

[8] Stark, P. C., Ryan, L. M., McDonald, J. L., \& Burge, H. A. (1997). Using meteorologic data to predict daily ragweed pollen levels. Aerobiologia, 13(3), 177. DOI= http://doi:10.1007/bf02694505

[9] Clark, C. J., \& Cochrane, L. M. (1999). Physical activity and asthma. Current Opinion in Pulmonary Medicine, 5(1), 68. 
[10] [Meltzer, L. J., Ullrich, M., \& Szefler, S. J. (2014). Sleep Duration, Sleep Hygiene, and Insomnia in Adolescents with Asthma. The Journal of Allergy and Clinical Immunology. In Practice, 2(5), 562-569. DOI= http://doi.org/10.1016/j.jaip.2014.02.005

[11] Tang, M., Agrawal, P., \& Jain, R. (2015). Habits vs Environment: What Really Causes Asthma? , 1-5. DOI= http://doi:10.1145/2786451.278648

[12] Kriksciuniene, D. P., Thomas; Kucera, Adam; Sakalauskas, Virgilijus. (2014). Data Analysis in the Intelligent Building Environment. International Journal of Computer Science and Applications, 11(1), 117.

[13] Castellsague, J., Sunyer, J., Sáez, M., \& Antó, J. M. (1995). Short-term association between air pollution and emergency room visits for asthma in Barcelona. Thorax, 50(10), 1051-1056. http://doi:10.1136/thx.50.10.1051

[14] Asthma Control iPhone App | AsthmaMD. Asthmam.org, 2016. Retrieved 25 February 2017, from http://www.asthmamd.org/

[15] Mack, H. (2016). Sparo Labs gets FDA nod for its app-connected lung function monitor, Wing. MobiHealthNews. Retrieved 25 February 2017, from http://www.mobihealthnews.com/content/sparo-labs-getsfda-nod-its-app-connected-lung-function-monitor-wing

[16] Ra, H.-K., Stankovic, J. A., Salekin, A., Yoon, H. J., Kim, J., Nirjon, S., . . . Son, S. H. (2015). Asthma Guide: An Ecosystem for Asthma Monitoring and Advice. 451-452.http://doi:10.1145/2809695.2817849

[17] Sofuoglu, S. C. (2008). Application of artificial neural networks to predict prevalence of building-related symptoms in office buildings. Building and Environment, 43(6), 1121-1126. http://dx.doi.org/10.1016/j.buildenv.2007.03.003

[18] Lewis, R. J. (2000). An introduction to classification and regression tree (CART) analysis. Paper presented at the Annual Meeting of the Society for Academic Emergency Medicine, San Francisco, California
[19] Gordon, L. (2013). Using Classification and Regression Trees (CART) in SAS $®$ Enterprise MinerTM For Applications in Public Health. University of Kentucky, Lexington, KY.

[20] How many people have an asthma action plan? (AIHW) Aihw.gov.au, 2016. http://www.aihw.gov.au/asthma/action-plan/.

[21] The National Asthma Council Australia. NationalAsthma.org.au, 2016. Retrieved 8 October 2016, from https://www.nationalasthma.org.au/living-with-asthma/asthmaaction-plans

[22] E. Jarauta-Bragulat, C. Hervada-Sala, and J. J. Egozcue, "Air Quality Index Revisited from a Compositional Point of View," Mathematical Geosciences, vol. 48, no. 5, pp. 581-593, 2016.

[23] [13] R. D. Ainsbury et al., "Method and apparatus for performing data collection, interpretation and analysis, in an information platform," ed: Google Patents, 2000.

[24] [14] W. Menke, Geophysical data analysis: discrete inverse theory: MATLAB edition. Academic press, 2012.

[25] N. G. O. o. E. Heritage. (2016, 10/13). Air Quality Index values. Available: http://www.environment.nsw.gov.au/aqms/aqitable.htm

[26] E. R. D. Interface. (n.d, 13/10). EIF Wiki. Available: https://eifwiki.feit.uts.edu.au/

[27] CAMA, J. 1998. Combination inhaler and peak flow rate meter. Google Patents.

[28] HUNTER, G. W., XU, J. C., BIAGGI-LABIOSA, A., LASKOWSKI, D., DUTTA, P., MONDAL, S., WARD, B., MAKEL, D., LIU, C. \& CHANG, C. 2011. Smart sensor systems for human health breath monitoring applications. Journal of breath research, 5, 037111.

[29] KANJO, E., BACON, J., ROBERTS, D. \& LANDSHOFF, P. 2009. MobSens: Making smart phones smarter. IEEE Pervasive Computing, 8.

[30] MCKENNA, M. 2013. The new age of medical monitoring. Scientific American, 308, 16-17.

APPENDIX

Available upon demand 